\title{
THE INDIRECT VERIFICATION OF THE GOING CONCERN ASSUMPTION BY ANALYSING THE THREAT OF BANKRUPTCY*
}

\author{
Michal Karas ${ }^{* *}$ \\ Mária Režñáková***
}

Received: 1. 9.2019

Preliminary communication

Accepted: 13. 10. 2020

UDC 658.14:330.131.7

DOI: https://doi.org/10.30924/mjcmi.25.2.2

\begin{abstract}
The generally accepted aim of doing business is to maximize the business value. The value of a business strongly depends on its ability to generate future cash flow for its owners. A necessary condition is that the business remains financially viable or in other words, the business should meet the assumption of going concern principle. Verifying such an assumption, however, remains an issue. We suggest that this could be indirectly verified as an absence of threat of imminent financial distress. For this purpose, we analysed

selected, due to their specific features resulting from the financial constraints. The F-test and $t$ test were employed as a method of identifying the typical signs of risk of financial distress or rather obstacles in accepting the going concern principle. We found that only four of the analysed ratios could serve effectively for this purpose. The best results were achieved when employing ratios describing the operational cash flow or short-term debt, where the gap between viable business and those with limited prospects is the widest.
\end{abstract} a set of cash flow and profit based ratios along with a set of other ratios with a potential influence on the business value. In terms of sample we focus on Czech manufacturing SMEs that were

Keywords: cash flow; going concern; business default

\section{INTRODUCTION}

According to the neoclassical theory of economics, the aim of doing business is to maximize the business value (see Damodaran, 2010; Brigham, Ehrhardt, 2010; McKinsey, Koller, Goedhart,

Wessels, 2005). This generally accepted idea has found its reflection in the concept of "value-based management" (further referred to as VBM). Knight (1998) defines the VBM "as an approach, which contributes to connecting the corporate

\footnotetext{
"A previous version of this paper has been presented and discussed at the 13th International Conference "Challenges of Europe: Growth, Competitiveness, Innovation and Well-Being”, organized by Faculty of Economics, Business and Tourism Split, in May 2019. Management - Journal of Contemporary Management Issues is one of the supporting journals of the Conference. All the papers have been reviewed and revised, according to the standards of this Journal.

** Michal Karas, PhD, assistant professor, Brno University of Technology, Faculty of Business and Management, Institute of Finance, Kolejní 2906/4, Královo Pole, 61200, Brno, Czech Republic, Phone: +420 54114 3708, Email: karas@fbm.vutbr.cz, ORCID: https://orcid.org/0000-0001-8824-1594

**** Mária Režňáková, PhD, professor, Brno University of Technology, Faculty of Business and Management, Institute of Finance, Kolejní 2906/4, Královo Pole, 61200, Brno, Czech Republic, Phone +420 54114 2790, E-mail: reznakova@fbm.vutbr.cz, ORCID: https://orcid.org/0000-0002-7261-607X
} 


\section{Journal of Contemporary Management Issues}

strategy, performance measurement and other activities contributing the maximization of the business value" (see also Karas, Režňáková, 2017). Similar definition could be found in Koller (1994) who describes VBM as a connection between thought contributing to increasing the business value and the corporate performance management systems. Frigo (2002) understands the VBM as "a tool of strategical performance measurement, which encourages the corporate management to focus on internal performance and by that support the business value creation".

Fourie (2010) summarizes the key features, distinguishing the VBM from other managerial approaches:

1. The aim of the value-based management is to contribute to the business value.

2. Value-based management identifies the so-called value drivers of the business.

3. VBM connects performance measuring and goal setting and it encourages the business value creation.

4. VBM combines decision-making and planning of value creating activities.

According to Pohl (2017,) the objective of value-based management is "to maximise a company's value. To achieve this, every management decision must aim to increase this value. This is the ultimate decision criterion that governs whether potential investments are realised, and if so, in which order of priority." To calculate a company's value for purposes of valuebased management, the following formula is used (Pohl, 2017):

$$
C V=\sum_{t=1}^{T} \quad \frac{C F_{t}}{(1+k)^{t}}+\frac{T V_{t}}{(1+k)^{T}},
$$

where: CV - company's value; T - time horizon; $\mathrm{CF}_{\mathrm{t}}$ - cash flow to equity at time $\mathrm{t} ; \mathrm{k}$ cost of capital; $\mathrm{TV}_{\mathrm{T}}$ - terminal value at time $\mathrm{T}$.

For a practical application of VBM, it is vital to identify the variables, which affect the cash flow to equity in the long term, or rather which affect the company's value either positively or negatively. Such variables are referred to as value drivers. Rappaport (1998), Copeland, Koller, Murrin (2005), Scarlett (2001) and Akalu (2002) define value drivers as "any variable affecting the value of a business". A similar view could be found in Koller, Goedhart and Wessels (2010), according to whom the value drivers are any activities, which affect the business performance (both in long-term and shortterm horizon) and by that, affect the value of the business. Finally, Woodcock (1992) describes value drivers as "any internal or external mechanisms, which are able to create or destroy the value of the business".

There is abundant research on value creating drivers. For example, Hall (2002) investigated the importance of given value drivers. As a result of his research, the following ranking of the value drivers is obtained (in order of their importance):

1. Return On Capital Employed (ROCE),

2. Weighted Average Cost of Capital (WACC),

3. Short-term liabilities divided by Capital Employed,

4. Debt ratio (total liabilities divided by Capital Employed),

5. Net Operating Profit Before Taxes (NOPBT) divided by Capital Employed,

6. Tax rate. 
Needles, Frigo and Powers (2004) explore the relationship between corporate strategy and corporate financial performance. According to their results, companies usually focus on indicators as total assets turnover, profit margin, debt-equity ratio and the ratio of operating cash flow and Earnings After Taxes (EAT). Under the concept of VBM, it is necessary to measure the value and, consequently, to focus on the identification of the key value drivers and affect them to achieve the aims of the owners or reditors. In both theory and practice, the model of Discounted Free Cash Flow (further referred to as FCF model) is most often employed for this purpose. The vital assumption of the FCF model application is meeting the going concern principle. In practice, this means that for the company to achieve its goal, it is necessary to fulfil the conditions for long-term growth. On the one hand, the company has to meet the customers' needs and constantly innovate its products and invest into technologies, while on the other hand, the financial stability of the business has to remain undisturbed, i.e. the solvency of the company has to be ensured and the risk, resulting from the high level of debt financing, should be minimized.

However, there is a practical question of how this assumption should be verified. Given that a company is considered to be "operational", if it is not directly threatened by bankruptcy, for this purpose, we suggest employing the bankruptcy prediction models, using the financial ratios. Moreover, as the value of a company is given by the FCF model, we assume that the cash flowbased indicators (such as Debt/operating cash flow), might be especially useful for this purpose. This is the reason why we focused on the prediction ability of indicators with a potential impact on business value, i.e. indicators that could be regarded as the value drivers.

\section{CAUSES OF CORPORATE BANKRUPTCY}

According to $\mathrm{Wu}$ (2010), the internal enterprise causes of distress "may be seen in insufficient management skills, marketing, and inability to compete". These features are reflected in financial performance of business and recorded in the financial statements, which is why the accounting ratios are a frequent source of information for assessing the financial situation of an enterprise. The companies undergoing business crises can be categorised as follows (Chen \& Hsiao, 2008):

- Companies lacking capital for running the business and starting having problems meeting their short-term monetary obligations (Deakin, 1972; Gilson, 1989), which is detectable in the values of current liquidity, quick liquidity, accounts receivable, cash flow, total asset turnover, and other factors.

- Companies with a negative value of retained profit for two consecutive periods, or a negative growth for at least 1 year. This is mainly evident from the following indicators: asset profitability, sales receipts, profits before and after taxes, and operating profit margin (Altman, 1983).

- Companies whose shares on a public stock market exhibit an overall drop or are even excluded from trading.

Lin, Liang, Chen (2011) noticed that the early studies mostly relied on the financial ratios of profitability, liquidity and solvency as indicators of financial difficulties, but the authors believe that further studies are needed, because there is research that has 


\section{Journal of Contemporary Management Issues}

indicated different ratios as being more reliable. Likewise, in different periods of the business cycle, we can identify different indicators of a firm's probability of distress (Mensah, 1984; Grice \& Dugan, 2001). According to Boratyńska (2016), there is a link between corporate distress (bankruptcy) and the business cycle, but "there is no agreement on the channels by which bankruptcies and the business cycle interact, nor on how to measure the link between them".

\section{INTERACTION BETWEEN CASH FLOW GENERATING ABILITY AND THREAT OF DEFAULT}

The interaction between cash flow generating ability and the business default is especially significant in case of SMEs. SMEs are often regarded as more constrained in their financing options than large businesses. Beck, Demirguc-Kunt, Laeven, Maksimovic (2006) describe a business as a financially constrained, "if a windfall increase in supply of internal funds results in a higher level of investment spending". A lot of research efforts have been dedicated to estimating financial constraints of firms (see e.g. Beck, Demirguc-Kunt, Laeven, Maksimovic, 2006; Fauceglia, 2015; Ullah, 2019; Erdogan, 2018; McGuinness, Hogana, Powell, 2018). Fazzari, Hubbard, Petersen (1988) showed that the investment decisions of financially constrained firms are more influenced by the availability of internal cash flows, when compared to the situation of unconstrained firms. Erdogan (2018) concluded that there is a relationship between a firm's growth potential and the limited internal funds, which, in turn, hinders their ability to make investments, causing obstacles to the growth of SMEs as a consequence. On a sample of French manufacturing firms, Musso and Schiavo (2008) show that financial constraints present a significant factor, driving a firm's survival, even after controlling for the effects of size, age, profitability and productive efficiency. McGuinness, Hogana, Powell (2018) examined whether the trade credit helped financially constrained SMEs survive the recent financial crisis, while above that highlights SMEs dependency on bank finance and their vulnerability to financing constraints (as noted by Beck, Demirguc-Kunt, Laeven, Maksimovic., 2006 or Stiglitz and Weiss, 1981). McGuinness, Hogana, Powell (2018) further add that the SMEs survival can depend on extension of additional trade credit and/or relax payment terms by their unconstrained creditors.

According to Jin, Luo, Wang (2018) businesses experiencing "higher financing constraints are more dependent on external funds and thus more sensitive to fluctuations in credit markets". North, Baldock, Ekanem (2010) point out that the main source of external finance to SMEs are the commercial banks.

From the above-mentioned perspective, the analysis of cash flow generating ability relatively to firm size (approximated by sales or total assets) or debt-repayment ability (approximated by various types of cash flow over liabilities) might gain an insight into the business ability of meeting the going concern principle. The aim of this paper is to analyse the potential of given financial ratios in predicting the corporate distress or rather as factors of destroying the business value of SMEs.

\section{SAMPLE AND METHODS USED}

The analysis was performed on a balanced sample of 88 Czech SMEs, out of which 44 companies were defaulted (bankrupt) and 44 non-defaulted. Applying a 
balanced sample could be viewed as a traditional approach in studies on corporate bankruptcy (see Beaver, 1966; Altman, 1968) although there are also many studies that use samples, with the proportion similar to the one in population (see for example Brezigar-Masten, Masten, 2012). The analysed companies were operating in the branch of manufacturing (NACE 2 section $\mathrm{C}$ ). The data were obtained from AMADEUS (Analysis Major Database for European Sources). The last report on defaulted companies dates to the period between 2013 and 2018. The reason for focusing on SMEs is their vulnerability in periods of economic recession, which is more strongly pronounced than in large or multinational companies (see Jin, Luo, Wang, 2018).

The significance of variables was tested by a two sample t-test, with equal or rather unequal variances. The test procedure can be described in the following manner. Let us suppose that we have two independent random samples $\left(X_{1}, \ldots, X_{n}\right)$ from distribution $\mathrm{N}\left(\mu_{1} \sigma^{2}\right)$, or rather $\left(Y_{1}, \ldots, Y_{m}\right)$ from distribution $\mathrm{N}\left(\mu_{2 ;} \sigma^{2}\right)$. Let's further assume that $n \geq 2 ; m \geq 2 ; \sigma^{2}>0$. The t-test assesses a null hypothesis, that the difference between the means of both groups $\left(\mu_{1}, \mu_{2}\right)$ is equal to some constant $(\Delta)$, usually to zero $(\Delta=0)$, i.e.

$$
H_{0}: \mu_{1}-\mu_{2}=\Delta
$$

against the alternative hypothesis:

$$
H_{1}: \mu_{1}-\mu_{2} \neq \Delta
$$

The test criterion, under assumption of equal variances can be expressed by the following form:

$|T|=\left|\frac{\bar{X}-\bar{Y}-\Delta}{\sqrt{(n-1) \cdot S_{x}^{2}+(m-1) \cdot S_{y}^{2}}} \cdot \sqrt{\frac{m \cdot(n+m-2)}{n+m}}\right| \geq t_{n+m-2(\alpha)}$,

where: $\bar{X}, \bar{Y}, S_{x}^{2}, S_{y}^{2}$ are characteristics of the two random samples.

The test criterion, under assumption of unequal variances can be expressed by the following form:

$$
\frac{|\bar{X}-\bar{Y}|}{S} \geq \frac{v_{x} t_{n-1}(\alpha)+v_{y} t_{m-1}(\alpha)}{v_{x}+v_{y}}
$$

where:

$S_{x}=\frac{1}{n-1}\left(\sum_{i=1}^{n} X_{i}^{2}-n \bar{X}^{2}\right), S_{y}=\frac{1}{n-1}\left(\sum_{i=1}^{m} Y_{i}^{2}-m \bar{Y}^{2}\right)$

$S=\sqrt{\frac{S_{x}^{2}}{n}+\frac{S_{y}^{2}}{m}}, v_{x}=\frac{S_{x}^{2}}{n}, v_{y}=\frac{S_{y}^{2}}{m}$

\section{RATIOS SELECTED FOR ANALYSIS}

The following set of ratios was collected by reviewing the literature on bankruptcy prediction or value drivers. 


\section{Journal of Contemporary Management Issues}

Table 1. Indicators used for analysis

\begin{tabular}{|c|c|c|}
\hline Shortcut & Description & Source \\
\hline $\mathrm{CFO} / \mathrm{CL}$ & Operating cash flow ${ }^{1} /$ current liabilities & $\begin{array}{l}\text { Bhandari, Johnson-Snyder (2018), Thomas Ng, } \\
\text { Wong, Zhang (2011) }\end{array}$ \\
\hline $\mathrm{CFO} / \mathrm{INT}$ & $\begin{array}{l}\text { (Operating cash flow }{ }^{1}+\text { interest } \\
\text { expenses }+ \text { tax }) / \text { interest expenses }\end{array}$ & Bhandari, Johnson-Snyder (2018) \\
\hline $\mathrm{CFO} / \mathrm{S}$ & Operating cash flow $1 /$ sales & $\begin{array}{l}\text { Bhandari, Johnson-Snyder (2018), Thomas Ng, } \\
\text { Wong, Zhang (2011) }\end{array}$ \\
\hline $\mathrm{CFO} / \mathrm{TA}$ & Operating cash flow $1 /$ total assets & $\begin{array}{l}\text { Bhandari and Johnson-Snyder (2018), Thomas Ng, } \\
\text { Wong, Zhang (2011) }\end{array}$ \\
\hline EBIT/CFO & EBIT/operating cash flow ${ }^{1}$ & Bhandari, Johnson-Snyder (2018) \\
\hline $\mathrm{CFO} / \mathrm{TL}$ & Operating cash flow ${ }^{1} /$ total liabilities $^{2}$ & Thomas Ng, Wong, Zhang (2011) \\
\hline $\mathrm{CF} / \mathrm{S}$ & Cash flow ${ }^{2} /$ sales & Beaver (1966) \\
\hline $\mathrm{CF} / \mathrm{TA}$ & Cash flow $2 /$ total assets & Beaver (1966) \\
\hline $\mathrm{CF} / \mathrm{TL}$ & Cash flow ${ }^{2} /$ total liabilities & Beaver (1966) \\
\hline $\mathrm{CF} / \mathrm{NW}$ & Cash flow ${ }^{2} /$ net worth & Beaver (1966) \\
\hline $\mathrm{CF} / \mathrm{CL}$ & Cash flow ${ }^{2} /$ current liabilities & Brezigar-Masten, Masten (2012) \\
\hline TL/TA & Total liabilities/total assets & $\begin{array}{l}\text { Brezigar-Masten, Masten (2012); Wang, Ma (2011), } \\
\text { Altman, Sabato (2006) }\end{array}$ \\
\hline $\mathrm{TA} / \mathrm{CL}$ & Total assets/ current liabilities & Brezigar-Masten, Masten (2012) \\
\hline $\mathrm{OR} / \mathrm{OC}$ & Operating revenue/operating cost & Brezigar-Masten, Masten (2012) \\
\hline $\mathrm{EAT} / \mathrm{TA}$ & Net profit/total assets & $\begin{array}{l}\text { Brezigar-Masten, Masten (2012); Cheng, Chen, Fu } \\
\text { (2006), Grunert, Norden, Weber (2004), Lin, (2009), } \\
\text { Wang, Lee (2008). }\end{array}$ \\
\hline EBIT/TA & Operating profit/total assets & $\begin{array}{l}\text { Alaminos, Del Castillo and Fernandez (2016); } \\
\text { Altman (1968); Li, Sun (2009); Psillaki, Tsolas, } \\
\text { Margaritis (2009); Shumway (2001) }\end{array}$ \\
\hline CA/TA & Current assets/Total assets & Alaminos, Del Castillo and Fernandez (2016) \\
\hline NWC/TA & Net Working Capital/Total assets & $\begin{array}{l}\text { Alaminos, Del Castillo and Fernandez (2016); } \\
\text { Altman (1968); Ding, Song, Zen(2008), Psillaki, } \\
\text { Tsolas, Margaritis (2009), Wu, Gaunt, Grey (2010) }\end{array}$ \\
\hline ROCE & EBIT/capital employed $^{3}$ & Hall (2002) \\
\hline $\mathrm{CL} / \mathrm{CE}$ & Current liabilities/capital employed ${ }^{3}$ & Hall (2002) \\
\hline $\mathrm{TL} / \mathrm{CE}$ & Current liabilities/capital employed ${ }^{3}$ & Hall (2002) \\
\hline $\mathrm{dNWC} / \mathrm{TA}$ & $\begin{array}{l}\text { Change of net working capital/total } \\
\text { assets }^{4}\end{array}$ & Minchington, Francis (2000); Rappaport (1998) \\
\hline $\mathrm{dNWC/S}$ & Change of net working capital $/$ sales $^{4}$ & Minchington, Francis (2000); Rappaport (1998) \\
\hline $\mathrm{dFA} / \mathrm{TA}$ & Change of fixed assets/total assets ${ }^{4}$ & Minchington, Francis (2000); Rappaport (1998) \\
\hline $\mathrm{dFA} / \mathrm{S}$ & Change of fixed assets/ sales ${ }^{4}$ & Minchington, Francis (2000); Rappaport (1998) \\
\hline
\end{tabular}


Source: Alaminos, Del Castillo,Fernandez (2016); Altman, Sabato (2006); Altman (1968); Beaver (1966); Bhandari, Johnson-Snyder (2018); Brezigar-Masten, Masten (2012); Masten (2012); Cheng, Chen, Fu (2006), Grunert, Norden, Weber (2004); Lin (2009), Wang, Lee (2008); Lin (2009); Wu, Gaunt, Grey (2010); Thomas Ng, Wong, Zhang (2011); Hall (2002); Minchington, Francis, (2000); Rappaport, (1998)

Note: ${ }^{1}$ Operating cash flow was calculated as EBIT + depreciation - [working capital $(t)$ working capital ( $t-1)] ;{ }^{2}$ Cash flow $=$ Net profit + depreciation; ${ }^{3}$ long-term liabilities + shareholder funds; ${ }^{4}$ The indicators is modified, in contrary to mentioned literature, to the form of ratio (i.e. divided by total assets or rather sales)

Included ratios are seen as being related to the value of the company, viewed from the perspective of FCF method, i.e. the majority of analysed ratios are cash flow- or profit-based (applying either cash flow or operating cash flow or net profit or operating profit), as the profit or rather cash flow generating ability has a direct influence on free cash flow and, thus, the business value. Other indicators describe the capital structure (in terms of indebtedness or capital intensity ratios) or describe the asset structure or investments (either in working capital or fixed assets).

\section{RESULTS}

The descriptive statistics of the continuous variables of the models under investigation are listed in the Table 2 . Several variables clearly exhibit an outlier's value, which is evident from the comparison of mean value and the median value. This situation is especially obvious in case of Cash flow coverage of interest $(\mathrm{CFO}+\mathrm{INT}+\mathrm{TAX}) / \mathrm{INT})$, while this situation is more obvious in case of non-failed companies, than in case of the failed companies. Among the specifics of failed companies in the sample, we can find features like negative operating cash flow (see indicators: $\mathrm{CFO} / \mathrm{CL}, \mathrm{CFO} / \mathrm{S}$ or $\mathrm{CF} / \mathrm{CL}$ ), negative Operating profit (see EBIT/TA) or a high level of issued debt (TL/TA indicator, which is a ratio of total liabilities and total assets). About $50 \%$ of the failed companies, financed at least $84 \%$ of their assets by debt, while in case of the non-failed companies, it was only $50 \%$ of assets.

Table 2. Descriptive statistics of the sample

\begin{tabular}{|l|l|r|r|r|r|r|r|}
\hline Variable & status & Valid N & \multicolumn{1}{l}{ Mean } & \multicolumn{1}{l}{ Median } & Min. & Max. & Std.Dev. \\
\hline $\begin{array}{l}\text { (CFO+INT } \\
\text { +TAX)/INT }\end{array}$ & Non-Failed & 37 & 1207.63 & 26.34 & -116.759 & 35028.00 & 5760.188 \\
\cline { 2 - 8 } & Failed & 10 & 21.60 & 7.57 & 0.31 & 119.338 & 36.352 \\
\hline \multirow{2}{*}{ CFO/CL } & Non-Failed & 42 & 0.49 & 0.44 & -0.651 & 1.55 & 0.519 \\
\cline { 2 - 9 } & Failed & 16 & -0.08 & 0.08 & -3.60 & 0.935 & 1.024 \\
\hline \multirow{2}{*}{ CFO/SALES } & Non-Failed & 43 & 0.08 & 0.08 & -0.488 & 0.28 & 0.128 \\
\cline { 2 - 8 } & Failed & 16 & -3.00 & 0.03 & -48.70 & 0.745 & 12.192 \\
\hline \multirow{2}{*}{ CFO/TA } & Non-Failed & 43 & 0.10 & 0.11 & -0.463 & 0.43 & 0.148 \\
\cline { 2 - 8 } & Failed & 16 & 30.73 & 0.06 & -6.68 & 498.286 & 124.693 \\
\hline \multirow{2}{*}{ EBIT/CFO } & Non-Failed & 43 & 0.48 & 0.48 & -1.911 & 3.36 & 0.928 \\
\cline { 2 - 8 } & Failed & 16 & -1.12 & 0.15 & -12.17 & 1.102 & 3.396 \\
\hline
\end{tabular}


Journal of Contemporary Management Issues

\begin{tabular}{|c|c|c|c|c|c|c|c|}
\hline \multirow{2}{*}{$\mathrm{CF} / \mathrm{CL}$} & Non-Failed & 42 & 0.41 & 0.31 & -0.454 & 1.41 & 0.364 \\
\hline & Failed & 18 & -1.04 & 0.01 & -15.34 & 0.285 & 3.673 \\
\hline \multirow{2}{*}{$\mathrm{TA} / \mathrm{CL}$} & Non-Failed & 42 & 3.96 & 3.25 & 1.050 & 12.38 & 2.487 \\
\hline & Failed & 27 & 1.56 & 1.34 & 0.00 & 3.667 & 1.020 \\
\hline \multirow{2}{*}{$\mathrm{OR} / \mathrm{OC}$} & Non-Failed & 43 & 1.05 & 1.04 & 0.648 & 1.15 & 0.079 \\
\hline & Failed & 26 & 0.91 & 1.01 & 0.02 & 1.123 & 0.251 \\
\hline \multirow{2}{*}{$\mathrm{TL} / \mathrm{TA}$} & Non-Failed & 43 & 0.47 & 0.50 & 0.084 & 0.98 & 0.250 \\
\hline & Failed & 27 & 66.84 & 0.84 & 0.27 & 1648.857 & 317.077 \\
\hline \multirow{2}{*}{ NWC/TA } & Non-Failed & 43 & 0.32 & 0.34 & -0.058 & 0.81 & 0.173 \\
\hline & Failed & 27 & -3.63 & 0.12 & -100.99 & 0.757 & 19.461 \\
\hline \multirow{2}{*}{ EBIT/TA } & Non-Failed & 43 & 0.07 & 0.07 & -0.300 & 0.29 & 0.092 \\
\hline & Failed & 27 & -224.86 & 0.00 & -6063.00 & 0.324 & 1166.764 \\
\hline \multirow{2}{*}{$\mathrm{CA} / \mathrm{TA}$} & Non-Failed & 43 & 0.62 & 0.62 & 0.221 & 0.94 & 0.202 \\
\hline & Failed & 27 & 0.66 & 0.72 & 0.00 & 1.000 & 0.304 \\
\hline \multirow[b]{2}{*}{$\mathrm{TL} / \mathrm{CE}$} & Non-Failed & 43 & 1.39372 & 0.746565 & 0.0919 & 20.10222 & 3.16675 \\
\hline & Failed & 27 & 6.926 & 1.156337 & -8.73 & 98.36905 & 21.722 \\
\hline \multirow[b]{2}{*}{ ROCE } & Non-Failed & 43 & -0.02166 & 0.099319 & -6.3233 & 0.54269 & 0.99278 \\
\hline & Failed & 27 & 0.87 & 0.095445 & -2.04 & 11.39968 & 2.667 \\
\hline \multirow[b]{2}{*}{$\mathrm{CFO} / \mathrm{TL}$} & Non-Failed & 43 & 0.344642 & 0.249879 & -0.5758 & 1.53015 & 0.48383 \\
\hline & Failed & 16 & 0.018 & 0.074998 & -0.9 & 0.58413 & 0.404 \\
\hline \multirow[b]{2}{*}{ CFO/EBIT } & Non-Failed & 43 & -0.32481 & 1.37294 & -70.6061 & 14.343 & 12.90439 \\
\hline & Failed & 16 & -1.533 & 1.521279 & -107.41 & 43.75 & 30.178 \\
\hline \multirow[b]{2}{*}{ dNWC/TA } & Non-Failed & 43 & 0.022106 & 0.019847 & -0.3479 & 0.57116 & 0.1486 \\
\hline & Failed & 23 & -375.41 & 0 & -8634.57 & 0.75656 & 1800.434 \\
\hline \multirow[b]{2}{*}{$\mathrm{dNWC/S}$} & Non-Failed & 43 & 0.004163 & 0.012866 & -0.2921 & 0.32134 & 0.1035 \\
\hline & Failed & 22 & -0.559 & -0.00411 & -12.91 & 0.85508 & 2.769 \\
\hline \multirow[b]{2}{*}{ dFA/TA } & Non-Failed & 43 & 0.015068 & 0.000536 & -0.1071 & 0.23832 & 0.0659 \\
\hline & Failed & 23 & -180.536 & 0 & -4154.29 & 0.59613 & 866.247 \\
\hline \multirow[b]{2}{*}{$\mathrm{dFA} / \mathrm{S}$} & Non-Failed & 43 & 0.007464 & 0.000273 & -0.1183 & 0.17369 & 0.05119 \\
\hline & Failed & 22 & -0.021 & 0.001495 & -6.21 & 4.29545 & 1.666 \\
\hline \multirow[b]{2}{*}{$\mathrm{CL} / \mathrm{CE}$} & Non-Failed & 43 & 1.183837 & 0.441091 & 0 & 20.10222 & 3.15277 \\
\hline & Failed & 27 & 7.283 & 0.798122 & -6.16 & 98.36905 & 21.488 \\
\hline \multirow[b]{2}{*}{$\mathrm{CF} / \mathrm{TL}$} & Non-Failed & 42 & 0.23533 & 0.248146 & -2.5445 & 1.25289 & 0.58196 \\
\hline & Failed & 15 & -0.836 & 0.001082 & -14.04 & 1.48299 & 3.685 \\
\hline \multirow[b]{2}{*}{$\mathrm{CF} / \mathrm{TA}$} & Non-Failed & 42 & 0.112535 & 0.097151 & -0.5921 & 0.55774 & 0.17737 \\
\hline & Failed & 15 & -21.533 & 0.001561 & -306.43 & 1.31104 & 78.894 \\
\hline \multirow[b]{2}{*}{$\mathrm{CF} / \mathrm{S}$} & Non-Failed & 42 & 0.079418 & 0.076853 & -0.2549 & 0.33234 & 0.08793 \\
\hline & Failed & 14 & -0.05 & 0.008337 & -1.94 & 1.48178 & 0.694 \\
\hline
\end{tabular}


Management, Vol. 25, 2020, No. 2, pp. 25-40 M. Karas, M.Režňáková: THE INDIRECT VERIFICATION OF THE GOING CONCERN...

\begin{tabular}{|l|l|l|l|l|l|l|l|}
\hline \multirow{3}{*}{ CF/NW } & Non-Failed & 42 & 0.983253 & 0.158793 & -0.7716 & 28.87624 & 4.42766 \\
\cline { 2 - 8 } & Failed & 15 & -10.649 & 0.032439 & -163.96 & 11.30705 & 42.576 \\
\hline
\end{tabular}

Source: Own calculation, based on the Amadeus database

For testing the usefulness of the indicators for predicting the corporate distress, we employed Student's t-test. According to the results of F-test the variance in both samples is different, thus the test criterion, under assumption of unequal variances has to be interpreted (see $t$ separ:; and corresponding p-value marked as $p(2))$.

Table 3. F-test and t-test results

\begin{tabular}{|c|c|c|c|c|c|c|c|c|}
\hline Variable & t-value & df & p-val.(1) & t separ. & df & p-val.(2) & F-ratio & p-val.(3) \\
\hline $\begin{array}{l}(\mathrm{CFO}+\mathrm{INT}+ \\
\mathrm{TAX}) / \mathrm{INT}\end{array}$ & -0.646 & 45 & 0.521624 & -1.252 & 36.011 & 0.218511 & 25108 & 0.000000 \\
\hline $\mathrm{CFO} / \mathrm{CL}^{* *}$ & -2.788 & 56 & 0.007240 & -2.111 & 18.019 & 0.048973 & 4 & 0.000548 \\
\hline CFO/SALES & -1.682 & 57 & 0.098010 & -1.011 & 15.001 & 0.328076 & 9109 & 0.000000 \\
\hline $\mathrm{CFO} / \mathrm{TA}$ & 1.635 & 57 & 0.107514 & 0.983 & 15.000 & 0.341388 & 706916 & 0.000000 \\
\hline EBIT/CFO & -2.846 & 57 & 0.006143 & -1.855 & 15.841 & 0.082336 & 13 & 0.000000 \\
\hline $\mathrm{CF} / \mathrm{CL}$ & -2.558 & 58 & 0.013163 & -1.671 & 17.143 & 0.112829 & 102 & 0.000000 \\
\hline $\mathrm{TA} / \mathrm{CL}^{* * *}$ & -4.749 & 67 & 0.000011 & -5.563 & 58.905 & 0.000001 & 6 & 0.000009 \\
\hline $\mathrm{OR} / \mathrm{OC}^{* *}$ & -3.390 & 67 & 0.001177 & -2.751 & 28.016 & 0.010291 & 10 & 0.000000 \\
\hline TL/TA & 1.379 & 68 & 0.172547 & 1.088 & 26.000 & 0.286745 & 1613080 & 0.000000 \\
\hline NWC/TA & -1.335 & 68 & 0.186436 & -1.053 & 26.003 & 0.302017 & 12702 & 0.000000 \\
\hline EBIT/TA & -1.270 & 68 & 0.208513 & -1.002 & 26.000 & 0.325702 & 159589462 & 0.000000 \\
\hline $\mathrm{CA} / \mathrm{TA}$ & 0.735 & 68 & 0.464917 & 0.671 & 40.442 & 0.505848 & 2 & 0.017326 \\
\hline $\mathrm{CFO} / \mathrm{EBIT}$ & -0.21671 & 57 & 0.829210 & -0.15494 & 17.08225 & 0.878687 & 5 & 0.000013 \\
\hline $\mathrm{CFO} / \mathrm{TL}^{* *}$ & -2.39970 & 57 & 0.019699 & -2.60667 & 31.99527 & 0.013769 & 1 & 0.457325 \\
\hline $\mathrm{CF} / \mathrm{S}$ & -1.20161 & 54 & 0.234759 & -0.69596 & 13.13953 & 0.498581 & 62 & 0.000000 \\
\hline $\mathrm{CF} / \mathrm{TA}$ & -1.80786 & 55 & 0.076098 & -1.06258 & 14.00005 & 0.305957 & 197848 & 0.000000 \\
\hline $\mathrm{CF} / \mathrm{NW}$ & -1.77249 & 55 & 0.081851 & -1.05612 & 14.10829 & 0.308663 & 92 & 0.000000 \\
\hline $\mathrm{CF} / \mathrm{TL}$ & -1.84967 & 55 & 0.069736 & -1.12117 & 14.25012 & 0.280753 & 40 & 0.000000 \\
\hline dNWC/TA & -1.37676 & 64 & 0.173383 & -1.00004 & 22.00000 & 0.328164 & 146800129 & 0.000000 \\
\hline $\mathrm{dFA} / \mathrm{TA}$ & -1.37614 & 64 & 0.173574 & -0.99959 & 22.00000 & 0.328377 & 172798057 & 0.000000 \\
\hline $\mathrm{dNWC} / \mathrm{S}$ & -1.34210 & 63 & 0.184380 & -0.95367 & 21.03002 & 0.351085 & 716 & 0.000000 \\
\hline $\mathrm{dFA} / \mathrm{S}$ & -0.11188 & 63 & 0.911274 & -0.07947 & 21.02030 & 0.937408 & 1059 & 0.000000 \\
\hline ROCE & 1.98958 & 68 & 0.050660 & 1.66552 & 30.57664 & 0.106024 & 7 & 0.000000 \\
\hline $\mathrm{CL} / \mathrm{CE}$ & 1.83785 & 68 & 0.070453 & 1.46509 & 26.70461 & 0.154572 & 46 & 0.000000 \\
\hline $\mathrm{TL} / \mathrm{CE}$ & 1.64939 & 68 & 0.103680 & 1.31469 & 26.69565 & 0.199799 & 47 & 0.000000 \\
\hline
\end{tabular}

Note: *significant at $5 \%$ level, **significant at $1 \%$ level, p-val.(1) - p-value of the test statistics under assumption of equal variances, p-val. (2) - p-value of the test statistics under assumption of unequal variances, p-val. (3) - p-value of the F-test. Source: Own calculation based on the Amadeus database 


\section{Journal of Contemporary Management Issues}

For a practical implication of the gained results, a deeper insight into values of the significant ratios is needed. For that purpose, we employ rank statistics.

Table 4. Rank statistics of the significant ratios

\begin{tabular}{|l|l|c|c|c|}
\hline Variable & Group & Lower quartile & Median & Upper quartile \\
\hline \multirow{2}{*}{ CFO/TL } & Failed & -0.0501 & 0.0750 & 0.2487 \\
\cline { 2 - 5 } & Non-failed & 0.1158 & 0.2499 & 0.5662 \\
\hline \multirow{2}{*}{ OR/OC } & Failed & 0.9273 & 1.0051 & 1.0292 \\
\cline { 2 - 5 } & Non-failed & 1.0229 & 1.0419 & 1.0976 \\
\hline \multirow{2}{*}{ TA/CL } & Failed & 0.8377 & 1.3399 & 2.2529 \\
\cline { 2 - 5 } & Non-failed & 2.1902 & 3.2480 & 5.6044 \\
\hline \multirow{2}{*}{ CFO/CL } & Failed & -0.0568 & 0.0787 & 0.3757 \\
\cline { 2 - 5 } & Non-failed & 0.1442 & 0.4416 & 0.9156 \\
\hline
\end{tabular}

Source: Own calculation based on the Amadeus database

We use the quartiles for describing the differences between healthy companies (nonfailed) and financially distressed companies (failed). As demonstrated by the descriptive statistics (see Table 4), these two groups are not completely separate. Due to such overlap, there is, still, at least a minimum chance of risk of financial distress. For a practical assessment of the acceptance of going concern assumption, we suggest to treat the following range of values as recommendable for accepting the assumption. The minimum acceptable value would be represented by the upper quartile of failed companies, while the upper quartile of non-failed companies would serve as recommended or target value - the higher this value, the more acceptable the situation.

Table 5. Minimum acceptable and target values for verifying the going concern principle

\begin{tabular}{|c|c|c|}
\hline Variable & Min. & Target \\
\hline $\mathrm{CFO} / \mathrm{TL}$ & 0.2487 & 0.5662 \\
\hline $\mathrm{OR} / \mathrm{OC}$ & 1.0292 & 1.0976 \\
\hline $\mathrm{TA} / \mathrm{CL}$ & 2.2529 & 5.6044 \\
\hline $\mathrm{CFO} / \mathrm{CL}$ & 0.3757 & 0.9156 \\
\hline
\end{tabular}

Source: Own calculation based on the Amadeus database
For business to remain viable, it is important to be able to generate operating cash flow, which covers at least $24.87 \%$ of the total debt, or rather preferably $37.57 \%$ of its short-term debts. Moreover, the total assets should cover the value of short-term debts at least 2.25 times (equivalent to maximum of $44 \%$ short-term indebtedness) or, rather, favourably 5.6 times (equivalent to maximum of $17.8 \%$ short-term indebtedness). Furthermore, the operation revenue should exceed the operation costs at least by $2.92 \%$, while favourably by at least $9.76 \%$ ).

\section{DISCUSSION}

The aim of this paper is to analyse the potential of financial ratios in predicting the corporate distress, considering them as factors with potential to destroy the business value. The set of potential ratios was collected by the review of current literature on corporate distress prediction and literature on business valuation.

According to the t-test, most significant ratios exploit the value of current liabilities, one in the relationship to operating cash flow (see $\mathrm{CFO} / \mathrm{CL}$ or $\mathrm{CFO} / \mathrm{TL}$ ) 
or in relationship to total assets value (TA/ $\mathrm{CL}$ ). It is important that similar ratios were also considered (see CF/CL or CF/TL). The only difference was employing cash flow (i.e. net profit + depreciation), instead of operating cash flow (i.e. EBIT + depreciation - change of net working capital). The financial expenses, taxes and changes of net working capital seem to play an important role in predicting bankruptcy, while their relationship to free cash flow and, thus, the business value is known (see Hall, 2002).

As for the cash flow generating ability, for the financially healthy SMEs in our sample, it is typical that the operating cash flow is about $49.5 \%$ of its current liabilities (the median value), while for failing SMEs, the typical value is only $8 \%$. In case of total liabilities, the operating cash flow for the financially healthy SMEs is about $24.98 \%$, while in case of failing ones only $7.49 \%$. The poor ability of failing companies in generating cash flow to cover their debts is in line with previous research (for example Beaver, 1966). Beaver (1966) pointed out the ratio cash flow to total debt as the most significant ratio of his study (among 30 others ratios). This ration, when employed in our study (see CF/TL), was outperformed by the $\mathrm{CFO} / \mathrm{TL}$ or $\mathrm{CFO} / \mathrm{CL}$ ratio.

Further focus on failing SMEs characteristics have revealed that about $50 \%$ of the failed companies financed at least $84 \%$ of their assets by debt, while in case of the healthy companies, it was only $50 \%$ of assets. This suggests that the capital structure of failed companies was inappropriate, which finally led to the solvency problems, and, consequently, to their bankruptcy. A further support for this claim could be found, when comparing the data on failed and non-failed companies in the sample. At one hand, a difference in the asset structure (see CA/TA indicator) between failed and non-failed companies was non-significant, but on the other hand, the difference in the capital structure (see the TA/CL indicator) was significant, which suggests that the failed companies were not able to match the capital and the assets structure. This is in line with other authors' results, who consider the higher proportion of debt in the company's capital structure as an impending indication of financial distress (see Zavgren, 1985). The importance and stability of total debt over assets ratio, based on a large sample of the four countries of Visegrad group, was also confirmed by Kliestik, Vrbka and Rowland (2018).

The significance of indebtedness in distress prediction has been summarised by Psillaki, Tsolas, Margaritis (2009), who argue that the total amount of debt "is regularly used as an indicator of a company's ability to meet its long-term debt obligations and remain solvent." The severity of the debt problem is usually assessed in relation to the value of assets, or as the total debt, i.e. the ratio of total liabilities to total assets (TL/TA). This definition of indebtedness became part of a series of studies and models (Beaver, 1966; Deakin, 1972; Ohlson, 1980; Ding, Song, Zen, 2008; Psillaki, Tsolas, Margaritis, 2009; Shin, Han, 2001; Altman, 1983; Zavgren, 1985; Wang, Ma, 2011; Altman, Sabato, 2006; Carling, Jacobson, Linde, Roszbach, 2007). Although the ratio of total liabilities to total assets (TL/TA) was under investigation in this study, the results do not exhibit significant values. It is worth saying that our study focuses on SMEs. Many traditional sources of long-term finance are more accessible to larger, rather than small businesses (see Atrill, 2003, p. 212). Thus, the SMEs are more vulnerable in the case of an economic recession than large or multinational companies (see Jin, Luo, Wang, 2018). 


\section{Journal of Contemporary Management Issues}

Focusing on the cash flow ratios, the only significant ratio based on cash flow was the ratio of cash flow to current liabilities. In the pioneer work of Beaver (1966), who analysed 30 financial ratios and their ability to indicate the risk of financial distress, the ratio of cash flow to total debts was found to be most useful for this purpose.

The usefulness of cash flow based ratio was a subject to research of Henerby (1996), according to whom, it could serve as an early warning indicator (at least three years, prior to failure), but is inappropriate for a short-term prognosis (one year, prior to failure). Our study also suggests that cash flow indicators are especially useful in predicting financial distress or threat to the long term perspective of the business - especially those, based on operating cash flow.

For a practical assessment of the acceptance of going concern assumption, we suggested to use the rank statistics of significant ratios as a suitable measure for assessing the going concern principle. To minimize the overlap of values, we suggest to treat the upper quartile of failed companies' values as the minimum acceptable value, while the upper quartile for non-failed companies would serve as recommended or target value.

\section{CONCLUSION}

The aim of the paper was to analyse the ability of financial ratios to reflect the obstacles in long-term business perspective, or rather, the obstacle in accepting the going concern principle. When valuing a business, the principle of going concern is often employed, however, the question is how this assumption should be proved. We argue that a company's inability to face such obstacles in long-term business perspective could result in corporate bankruptcy and by evaluating the threat of bankruptcy, the assumption of going concern could be indirectly verified.

It was proved that such a threat is reflected in financial statements and by analysing financial ratios, such a threat could be evaluated. Our research focuses on SMEs that are often financially constrained, i.e. they have limited access to long-term sources of finance, compared to large companies. Because of that, their capital structure is different and the typical signs of bankruptcy threat could differ.

The results show that the majority of financial ratios under analysis did not exhibit sufficient explanatory power, in terms of reflecting the risk of bankruptcy or, rather, threatening the long-term prosperity of the business. In other words, verifying the assumption of going concern principle in case of SMEs, cannot be effectively done by focusing on the commonly employed financial ratios.

According to our data, the signs of poor future prospects of the SMEs are poor cash generating ability, especially in relation to its debts, as well as a high level of debt (especially short-term debts) and the level of operation cost.

\section{References}

1. Akalu, M. M. (2002). Measuring and Ranking Value Drivers [online]. Tinbergen Institute Discussion Paper]. Available at $\mathrm{z}$ http://repub.eur.nl/res/ pub/6815/2002-0432.pdf.

2. Alaminos, D., Del Castillo, A. \& Fernandez, M., A. (2016) A Global 
Model for Bankruptcy Prediction. Plos One. 11(11), pp. 1-18.

3. Altman, E. I. (1968). Financial Ratios, Discriminant Analysis and the Prediction of Corporate Bankruptcy. The Journal of Finance, 23(4), 589-609.

4. Altman, E. I. Sabato, G. (2006). Modelling credit risk for SMEs: Evidence from US market [online] http://people.stern.nyu.edu/ealtman/ ModelingCreditRiskforSMEs\%20.pdf

5. Altman, E. I. (1983). Corporate financial distress: A complete guide to predicting, avoiding and dealing with bankruptcy. New York: John Wiley and Sons.

6. Atrill. P. (2003). Financial management for Non-specialists. 3 ed. Prentice Hall.

7. Beaver, W. H. (1966). Financial Ratios as predictors of Failure. Journal of Accounting Research, 4, 71-111.

8. Beck, T., Demirguc-Kunt, A., Laeven, L., \& Maksimovic, V. (2006). The determinants of financing obstacles. Journal of International Money and Finance, 25, 932-952.

9. Bhandari, S., Johnson-Snyder, A. J., (2018). A Generic Model of Predicting Probability of Success-Distress of an Organization: A Logistic Regression Analysis. The Journal of Applied Business Research, 34(1), 169-182.

10. Boratyńska, K. (2016). Corporate Bankruptcy and Survival on the Market: Lessons from Evolutionary Economics, Oeconomia Copernicana, 7(1), 107-129.

11. Brezigar-Masten, A., Masten, I. (2012). CART-based selection of bankruptcy predictors for the logit model. Expert Systems with Applications, 39, 10153-10159
12. Brigham, E. R., Ehrhardt, M. C. (2010). Financial Management. Theory and Practice. South-Western College Pub.

13. Carling, Jacobson, Linde, Roszbach, K., Jacobson, T., Linde, J. \& Roszbach, K. (2007). Corporate credit risk modelling and the macroeconomy. Journal of Banking \& Finance, 31(3), 845-868.

14. Chen, L. H., Hsiao, H. D. (2008). Feature selection to diagnose a business crisis by using a real GA-based support vector machine: An empirical study. Expert Systems With Applications, 35, 1145-1155,

15. Cheng, C. B., Chen, C., L. \& Fu, C. J. (2006). Financial Distress Prediction by a Radial Basis Function Network with Logit Analysis Learning. Computers and Mathematics with Applications, 51, 579-588.

16. Copeland, T., Koller, T. \& Murrin, J. (2005). Valuation - Measuring and Managing the Value of Companies. New York: John Wiley and Sons.

17. Damodaran, A. (2010). The dark side of valuation: valuing young, distressed and complex businesses. New Jersey: Pearson Education.

18. Deakin, E. B. (1972). A Discriminant Analysis of Predictors of Business Failure. Journal of Accounting Research, 10 (1), 167-179.

19. Ding, Y., Song, X. \& Zen, Y. (2008). Forecasting financial condition of Chinese listed companies based on support vector machine. Expert Systems with Applications. 34, 3081-3089.

20. Erdogan, A. I., (2018). Factors affecting SME access to bank financing: An interview study with Turkish bankers. Small Enterprise Research, 25(1), 23-35. 


\section{Journal of Contemporary Management Issues}

21. Fazzari, S. M., Hubbard, R. G. \& Petersen, B. C. (1988) Financing Constraints and Corporate Investment. Brookings Papers on Economic Activity, 1, 141-195.

22. Fauceglia, D. (2015). Credit constraints, firm exports and financial development: Evidence from developing countries. The Quarterly Review of Economics and Finance, 55, 53-66.

23. Fourie, L. A. (2010). The value based management and share price relationship for companies listed on banking sector of the JSE Ltd. Potchefstroom (Mini- dissertation - MBA). NorthWest University.

24. Frigo, M. L. (2002). Strategy execution and value based management. Strategic Finance, 84(4), 6-9.

25. Gilson, S. C. (1989). Management turnover and financial distress. Journal of Financial Economics, 25 (2), 241-262.

26. Grice, J. S., Dugan, M. T. (2001). The limitations of bankruptcy prediction models: Some cautions for the researchers. Review of Quantitative Finance and Accounting, 17(2), 151-166.

27. Grunert, J., Norden, L. \& Weber, M. (2004). The role of non-financial factors in internal credit ratings. Journal of Banking \& Finance, 29 (2), 509-531.

28. Hall, J. H. (2002). Dissecting EVA: The value drivers determining the shareholder value of industrial companies [online]. Pretoria: University of Pretoria. http://www.fep.up.pt/disciplinas/mbf922/Dissecting\%20EVA.pdf.

29. Henerby, K. L. (1996). Do Cash Flows Variables Improve the Prediction Accuracy of a Cox Proportional Hazards Model for Bank Failure? The
Quarterly Review of Economics and Finance, 36(3), 395-409.

30. Jin, Y. Luo, M., Wan, C. (2018). Financial constraints, macro-financing environment and post-crisis recovery of firms. International Review of Economics and Finance, 55 (C), 54-67.

31. Karas, M., Režňáková, M. (2017). The stability of bankruptcy predictors in the construction and manufacturing industries at various times before bankruptcy. E\&M: Economics and Management, 20(2), 116-133.

32. Kliestik, T., Vrbka, J. \& Rowland, Z. (2018). Bankruptcy prediction in Visegrad group countries using multiple discriminant analysis. Equilibrium. Quarterly Journal of Economics and Economic Policy, 13(3), 569-593.

33. Knight, J. A. (1998). Value based management: developing a systematic approach to creating shareholder value. New York: McGraw-Hill.

34. Koller, T. (1994). What is value based management? McKinsey, Koller, Goedhart, Wessels Quarterly, 3, 87-101.

35. Koller, T., Goedhart, M. \& Wessels, D. (2010). Valuation. Measuring and managing the value of companies. New Jersey: John Wiley \& Sons.

36. Lin, F., Liang, D., Chen, E. (2011). Financial ratio selection for business crisis prediction. Expert Systems with Applications, 38, 15094-15102.

37. Lin, S. L. (2009). A new two-stage hybrid approach of credit risk in banking industry, Expert Systems with Applications, 36 (4), 8333-8341.

38. McGuinness, G., Hogana, T., Powell, R. (2018). European trade credit use and SME survival. Journal of Corporate Finance, 49, 81-103. 
39. McKinsey, Koller, Goedhart, Wessels \& Company, Koller, T., Goedhart, M., Wessels, D. (2005). Measuring and Managing the Value of Companies. New Jersey: Wiley and Sons.

40. Mensah, Y. M. (1983). The Differential Bankruptcy Predictive Ability of Specific Price Level Adjustments: Some Empirical Evidence. The Accounting Review, 58 (2), 228-246.

41. Minchington, C., Francis, G. (2000). Shareholder value. Management Quarterly, 6, 23-31.

42. Musso, P., Schiavo, S. (2008). The impact of financial constraints on firm survival and growth. Journal of Evolutionary Economics, 18(2), 135-149.

43. Needles, B. E., Frigo, M. L., Powers, M. (2004). Strategy and integrated financial ratio performance measures: Empirical evidence of the financial performance scorecard and high performance companies. Studies in Managerial and Financial Accounting, 14, 115 - 151 .

44. North,D., Baldock, R., Ekanem, I. (2010). Is there a debt finance gap relating to Scottish SMEs? A demandside perspective. Venture Capital, 12(3), 173-192.

45. Ohlson, J. A. (1980). Financial Ratios and the Probabilistic Prediction of Bankruptcy. Journal of Accounting Research, 18(1), 109-131.

46. Pohl, P. (2016). Valuation of a Company using Time Series Analysis. Journal of Business Valuation and Economic Loss Analysis, 12(1), 1-39.

47. Psillaki, M. Tsolas, I. T. \& Margaritis, M. (2010). Evaluation of credit risk based on firm performance, European Journal of Operational Research, 201, 873-881.
48. Rappaport, A. (1998). Creating shareholder value: A guide for managers and investors. New York: The Free Press.

49. Scarlett, R. C. (2001). Valuebased Management. London: Ivey Management Services.

50. Shin, K., Han, I. (2001). A case-based approach using inductive indexing for corporate bond rating, Decision Support Systems, 32, 41-52.

51. Stiglitz, J., Weiss, A. (1986). Credit rationing: Reply. American Economic Review, 77, 228-231.

52. Thomas Ng, S. T., Wong, J. M. W., Zhang, J. (2011). Applying Z-score model to distinguish insolvent construction companies in China. Habitat International, 35(1), 599-607.

53. Ullah, B. (2019). Financial constraints, corruption, and SME growth in transition economies. The Quarterly Review of Economics and Finance, 75(C), 120-132.

54. Wang, G., Ma, J. (2011). Study of corporate credit risk prediction based on integrating boosting and random subspace. Expert Systems with Applications, 38, 13871-13878.

55. Wang, Y. J., Lee, H. S. (2008). A clustering method to identify representative financial ratios. Information Sciences, 178, 1087-1097.

56. Woodcock, J. (1992). Buying or Selling a Business? Don't be Ripped Off. Business Quarterly, 57 (2), 41-47.

57. Wu, W. (2010). Beyond business failure prediction, Expert Systems with Applications, 37 (3), 2371-2376.

58. Wu, Y., Gaunt, C., Gray, S. (2010). A comparison of alternative bankruptcy prediction models. Journal 


\title{
Journal of Contemporary Management Issues
}

of Contemporary Accounting \& industrial firms: A logistic analyEconomics, 6, 34-45. sis. Journal of Business Finance and

59. Zavgren, C. V. (1985). Assessing the Accounting, 12 (1), 19-45. vulnerability to failure of American

\section{INDIREKTNA VERIFIKACIJA PRETPOSTAVKE TRAJNOG POSLOVANJA KORIŠTENJEM ANALIZE STEČAJNE PRIJETNJE}

\begin{abstract}
Sažetak
Opće prihvaćeni cilj poslovanja je maksimiziranje vrijednosti poduzeća, koja značajno ovisi o sposobnosti za stvaranje budućih tijekova novca za vlasnike. Pritom se podrazumijeva uvjet financijske održivosti poduzeća, odnosno ispunjavanje pretpostavke trajnosti poslovanja. Ipak, verifikacija navedene pretpostavke ostaje i dalje otvoreno pitanje. Smatramo da se isto može riješiti indirektnom verifikacijom, koja podrazumijeva nepostojanje prijetnje bliske financijske krize. U tu smo svrhu analizirali skupinu pokazatelja, zasnovanih na novčanom tijeku i dobiti, zajedno sa skupinom drugih pokazatelja, s potencijalnim djelovanjem na vrijednost poduzeća. Koncentriramo se na uzorak malih i srednjih proizvodnih poduzeća u Češkoj, a koja su izabrana, s obzirom na specifična obilježja, povezana s financijskim ograničenjima. Za identifikaciju tipičnih znakova rizika financijskih poteškoća, odnosno nepoštovanja pretpostavke trajnosti poslovanja, koriste se $F$ - $i$ t-test. Za efikasno korištenje u navedene svrhe, pozitivno smo vrednovali samo četiri od analiziranih pokazatelja. Najbolji se rezultati dobivaju korištenjem indikatora, koji opisuju operativni novčani tijek ili kratkoročni dug, kod kojih se javljaju najveće razlike između održivih poduzeća i onih s ograničenim perspektivama.
\end{abstract}

Ključne riječi: gotovinski tijek, pretpostavka trajnog poslovanja, stečaj 